\section{Implant Therapy in the Esthetic Zone Current Treatment Modalities and Materials for Single-tooth Replacements}

Authors: Daniel Buser / Stephen Chen / Daniel Wismeijer (Ed.)

Publisher: Quintessence Publishing, Germany

Language: English

ISBN: 978-3-86867-343-2

Edition: 1/e

Publish Year: 2017

Pages: 444, illustrated
Price: $86.00 €$

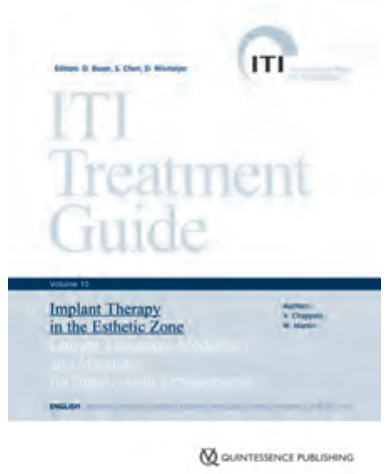

\section{Florin-Eugen \\ Constantinescu}

DMD, PhD Student

Holistic Dental \& Medical Institute of

Bucharest-ROPOSTURO, Bucharest

Romania

e-mail:

dr.florin.constantinescu@gmail.com

In current practice dental implants are used more and more frequently to replace missing teeth, whose therapeutic success can be ensured by applying treatment methods that meet the highest clinical standards.

The ITI Treatment Guide series written by well-known clinicians and practitioners is a compendium of evidence-based implant techniques and procedures for everyday practice.

Volume 10 of the ITI Treatment Guide series entitled "Implant Therapy in the Esthetic Zone" edited by Dr. Daniel Buser, Professor and Chair at the Department of Oral Surgery and Stomatology at the University of Bern, Dr. Stephen Chen, Clinical Associate Professor at the School of Dentistry at the University of Melbourne and Dr. Daniel Wismeijer, Professor of Oral Implantology and Prosthetic Dentistry at the Academic Center for Dentistry of Amsterdam (ACTA), presents current treatment modalities and materials for single tooth replacements. The book is divided in ten chapters. Following the discussion on the topic approached in the introductory chapter, the second chapter presents the statements and recommendations of the 5th ITI Consensus Conference. A protocol for evaluating and planning treatment for patients whose aesthetic requirements demand the replacement of a single tooth with a dental implant is presented in the following chapter. Next, we are told how to select biomaterials for implantation procedures and which are the surgical considerations for optimal aesthetic results in different surgical situations encountered in the aesthetic area. The sixth chapter deals with the prosthetic management for optimal esthetic outcomes starting from the clinical examination of the planned implant site before and after placement using temporary prostheses, communication with the dental laboratory, abutment design, restorative material selection, and delivery. A consistent chapter is allocated to clinical case presentations developing the restorations made in various surgical situations encountered in the aesthetic area. In the eighth chapter the authors honestly present the possible esthetic complications they faced. The penultimate chapter mentions the conclusions reached by the authors in the treatment of patients whose aesthetic requirements demanded the replacement of a single tooth with a dental implant.

This volume, based on the latest references in the specialty literature gathered in the last chapter, is consistently enriched with over 1380 compelling quality illustrations.

This volume of the ITI Treatment Guide series provides the reader with an evidence-based approach to replace teeth in the aesthetic area with dental implants, from consultation to follow-up, to the choice of biomaterials and surgical procedures leading to an optimal esthetic treatment outcome according to the most advanced possibilities offered by oral implantology today.

DOI: 10.25241/stomaeduj.2018.5(2).bookreview.5 